\title{
A Chinese Hamster Ovary Leucyl-tRNA Synthetase Mutant with a Uniquely Altered High Molecular Weight Leucyl-tRNA Synthetase Complex
}

\author{
Alka Mansukhani, ${ }^{1}$ Tom Condon, 'Arnold Hampel, ${ }^{1}$ and Dale L. Oxender ${ }^{2}$
}

Received 3 Aug. 1983-Final 30 Nov. 1983

The Chinese hamster ovary (CHO) cell culture temperature-sensitive mutant ts $025 \mathrm{Cl}$ with a defect in leucyl-tRNA synthetase (LeuRS) does not have an inherently more thermolabile LeuRS, but instead the mutation causes the complete loss of the LeuRS high molecular weight complexes which are present in normal wild-type cells. The mutant cell LeuRS has a single $8 \mathrm{~S}$ enzyme form which corresponds hydrodynamically to the $8 \mathrm{~S}$ free form of wild-type enzyme. Both $8 \mathrm{~S}$ forms have the same thermostability and the same $\mathrm{K}_{\mathrm{m}}$ for leucine, indicating that there is no inherent defect in the catalytic activity of the enzyme. The temperature-sensitive phenotype can be explained by the lack of thermostable high molecular weight forms of LeuRS.

KEY WORDS: leucyl-tRNA; Chinese hamster ovary enzyme complexes.

\section{INTRODUCTION}

The Chinese hamster ovary ( $\mathrm{CHO}$ ) cell line has proven useful for the isolation of temperature-sensitive conditionally lethal mutants (Thompson et al., 1973, $1975,1977)$. The mutant $t s 025 \mathrm{Cl}$ had a temperature-sensitive phenotype with low levels of LeuRS activity and was used to assign the location of the human leucyl-tRNA synthetase (LeuRS) genetic locus to chromosome 5 of the human genome (Giles et al., 1980). Others had previously shown that the

This work was supported by NIH Grant GM 19506 to A.E.H. and GM 20737 to D.E.O.

${ }^{1}$ Departments of Biological Sciences, Northern Illinois University, DeKalb, Illinois 60115.

${ }^{2}$ Department of Biological Chemistry, University of Michigan, Ann Arbor, Michigan 48109. 
mutation was recessive (McBurney and Whitmore, 1975), expressed a leucine phenotypic protective effect, and did not complement the well-characterized LeuRS mutant $t s \mathrm{H} 1$ (Thompson et al., 1975). The mutant $t s \mathrm{H} 1$ has a thermolabile LeuRS with low levels of enzyme activity (Thompson et al., 1973). The enzyme is kinetically altered (Haars et al., 1976; Farber and Deutscher, 1976), with a leucine $K_{m}$ about $4 \times$ that of wild type (WT) (Klekamp et al., 1981). When the defective LeuRS is analyzed on sucrose gradients, only the low molecular weight $8 \mathrm{~S}$ form of the enzyme is seen, while wild-type cells have three hydrodynamic forms of LeuRS-a low molecular weight form at $8 \mathrm{~S}$ and two high molecular weight forms at $20 \mathrm{~S}$ and $30 \mathrm{~S}$ which account for about $70 \%$ of the total LeuRS activity (Hampel et al., 1978; Ritter et al., 1976, 1979).

In this report we show that the temperature-sensitive LeuRS mutant $t s 025 \mathrm{Cl}$ does not have an inherently thermolabile LeuRS. Only the $8 \mathrm{~S}$ form of LeuRS exists, which has the same thermostability as wild-type $8 \mathrm{~S}$ and the same leucine $K_{m}$ as wild-type $8 \mathrm{~S}$. The reason for the $t s$ phenotype can be explained by the lack of thermostable high molecular weight complexes. These results are important in understanding which component of LeuRS was mapped on the human genome and in our eventual understanding of the nature and function of the high molecular weight aminoacyl-tRNA synthetase complexes commonly found in mammalian cells (Bandyopadhyay and Deutscher, 1971; Schimmel and Soll, 1979).

\section{MATERIALS AND METHODS}

\section{Size Distribution of Leucyl-tRNA Synthetase on Sucrose Gradients}

Chinese hamster ovary $(\mathrm{CHO})$ cells were grown as previously described (Klekamp et al., 1981). Cells $\left(10^{8}\right)$ were lysed with Nonidet-P40, nuclei and cell debris were removed, and supernatant $(0.7 \mathrm{ml})$ was layered on $12-\mathrm{ml}$ $10-30 \%(\mathrm{w} / \mathrm{v})$ linear sucrose gradients in $10 \mathrm{mM} \mathrm{KCl}, 1 \mathrm{mM} \mathrm{MgCl}, 0.1 \mathrm{mM}$

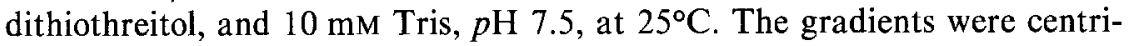
fuged in a Beckman SW 28.1 rotor at $28,000 \mathrm{rpm}$ for $16 \mathrm{hr}, 40 \mathrm{~min}$ at $4^{\circ} \mathrm{C}$, fractionated, and assayed for LeuRS activity using $\left[{ }^{3} \mathrm{H}\right]$ leucine as a substrate and counting $\left[{ }^{3} \mathrm{H}\right]$ Leu-tRNA ${ }^{\mathrm{Leu}}$ as a product of the reaction (Klekamp et al., 1981). Sedimentation coefficients $\left(s_{20, w}\right)$ were calculated on a computer program as previously described (Dingman, 1972). The total protein in $10^{8}$ cells was $35 \mathrm{mg}$ as determined by the method of Lowry et al. (1951).

\section{Thermostability of the $8 \mathrm{~S}$ Form of LeuRS from Three Cell Types}

Protein concentrations in tubes containing the $8 \mathrm{~S}$ LeuRS peaks from the sucrose gradients were determined and the protein was adjusted to $10 \mathrm{mg} / \mathrm{ml}$ 
by the addition of bovine serum albumin (Sigma). Purified rat liver tRNA was added to a final concentration of $3.0 \mathrm{mg} / \mathrm{ml}$ and the tubes were heated at $40.5^{\circ} \mathrm{C}$. Aliquots $(10 \mu \mathrm{l})$ were removed after $0,2,4,6,8,10,12$, and $14 \mathrm{~min}$ and assayed at $34^{\circ} \mathrm{C}$ for LeuRS activity in a $50-\mu$ l incubation mixture as described (Klekamp et al., 1981).

\section{Leucine Michaelis Constants of Wild-Type $8 \mathrm{~S}$ LeuRS and the Mutant ts $025 \mathrm{Cl} 8 \mathrm{~S}$}

Peak tubes from sucrose gradients were assayed for leucyl-tRNA synthetase activity over a range of leucine concentrations, $2,4,8,12,16$, and $20 \mu \mathrm{M}$, as described previously (Haars et al., 1976, Klekamp et al., 1981). Assay times of $0.5,1.0$, and $1.5 \mathrm{~min}$ gave increasing linear product formation with respect to time under the conditions employed. Double reciprocal plots $(1 / \mathrm{v}$ vs $1 / \mathrm{S})$ were analyzed by linear regression and the calculated $K_{m}$ values confirmed by standard statistical kinetic analysis (Wilkinson, 1961).

\section{RESULTS AND DISCUSSION}

The presence of only the low molecular weight form of the LeuRS in the CHO cell LeuRS mutant $t s 025 \mathrm{Cl}$ can be seen in Fig. 1. Wild-type cells show the three regular forms of the enzyme at $8 \mathrm{~S}, 20 \mathrm{~S}$, and $30 \mathrm{~S}$. This mutant profile shows no evidence of any high molecular weight LeuRS complexes and contains about $12 \%$ of wild-type LeuRS activity. The mutant $8 \mathrm{~S}$ LeuRS regularly moves one tube slower than wild-type LeuRS on sucrose gradients, a behavior which is very similar to that of the mutant $t s \mathrm{H} 1$ which we analyzed previously and indicates that the $8 \mathrm{~S}$ forms are not identical but that $t s 025 \mathrm{CI}$ $8 \mathrm{~S}$ is physically different than wild type.

The thermolability of the respective enzyme forms is shown in Fig. 2. The gradient tubes from the $8 \mathrm{~S}$ LeuRS peak of the sucrose gradient in Fig. 1 were heated at enzyme-inactivating temperature as described in Materials and Methods and were assayed for loss of LeuRS activity over time. Wild-type $8 \mathrm{~S}$ LeuRS and $t s 025 \mathrm{Cl} 8 \mathrm{~S}$ LeuRS showed the same thermolability. The mutant $t s \mathrm{H} 18 \mathrm{~S}$ LeuRS was run as a control and shows its typical temperature sensitivity, with a thermal inactivation rate about $3 \times$ that of wild-type $8 \mathrm{~S}$ (Klekamp et al., 1981). The thermostability of wild-type $30 \mathrm{~S}$ and $20 \mathrm{~S}$ LeuRS relative to wild-type $8 \mathrm{~S}$ has been previously published by us, and we showed that wild-type $20 \mathrm{~S}$ LeuRS has the same thermostability as $30 \mathrm{~S}$ LeuRS, with both being $2 \times$ more stable than wild-type $8 \mathrm{~S}$. We also showed that the greatest stability occurs when all three forms are incubated together. In the case of the mutant described here, however, only the most inherently thermolabile form $(8 \mathrm{~S})$ of LeuRS exists. The similarity between $t s 025 \mathrm{Cl} 8 \mathrm{~S}$ LeuRS and the wild-type $8 \mathrm{~S}$ form was further confirmed by leucine $K_{m}$ 




Fig. 1. Size distribution of leucyl-tRNA synthetase on sucrose gradients. LeuRS enzyme activity from wild type $(-)$ and mutant $t 5025 \mathrm{Cl}(-\mathrm{O}-$ ) CHO cells. The same number of cells $\left(10^{8}\right)$ and total protein $(35 \mathrm{mg})$ were added to each gradient. Further details are as in Materials and Methods.

determinations (Fig. 3). Both enzymes showed the same leucine $K_{m}$ of $16 \mu \mathrm{M}$, which is similar to that we previously published for wild-type $8 \mathrm{~S}$ (Klekamp et $a l ., 1981)$. The temperature-sensitive LeuRS mutant $t s 025 \mathrm{Cl}$ clearly contains an altered LeuRS, but the alteration affects the ability of the mutant LeuRS to form complexes and does not affect the catalytic activity of the free form $(8 \mathrm{~S})$ of the enzyme. The mutant clearly has only the $8 \mathrm{~S}$ form, with no traces of the higher molecular weight complexes found in wild-type cells. The respective $8 \mathrm{~S}$ forms from these cell types show the same thermolability and have the same leucine $K_{m}$. This is in contrast to the previously characterized CHO LeuRS $t s \mathrm{H} 1$, which has a very thermolabile $8 \mathrm{~S}$ form of the enzyme with a fourfold larger $K_{m}$ (Klekamp et al., 1981).

The question, then, What is the basis of the temperature-sensitive phenotype of $t s 025 \mathrm{Cl}$ ? is posed. We previously showed that the high molecular weight $20 \mathrm{~S}$ and $30 \mathrm{~S}$ LeuRS complexes of wild-type cells are $2 \times$ more 


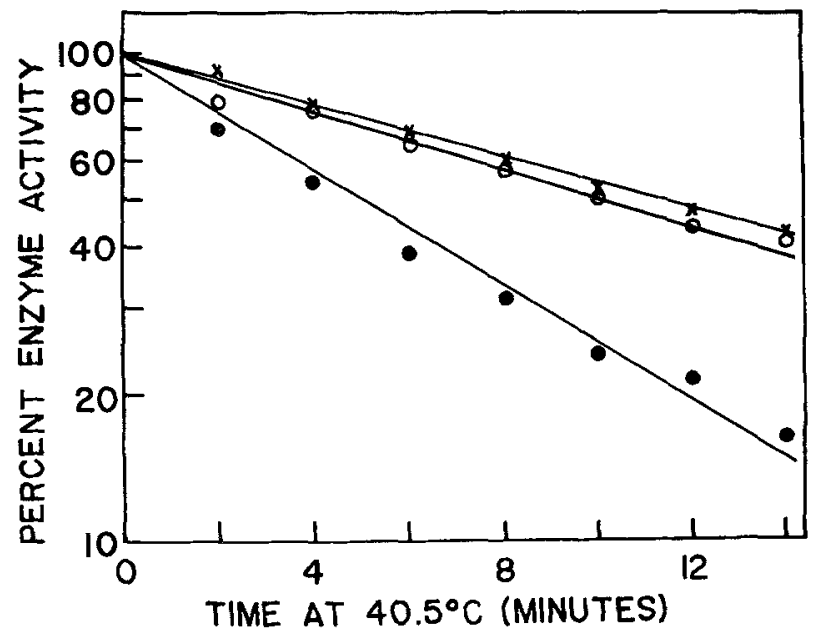

Fig. 2. Thermostability of the $8 \mathrm{~S}$ form of LeuRS from three cell types: wild type (-X-); LeuRS mutant $t s 025 \mathrm{Cl}(-\mathrm{O}-)$; and LeuRS mutant ts $\mathrm{H} 1(--)$.

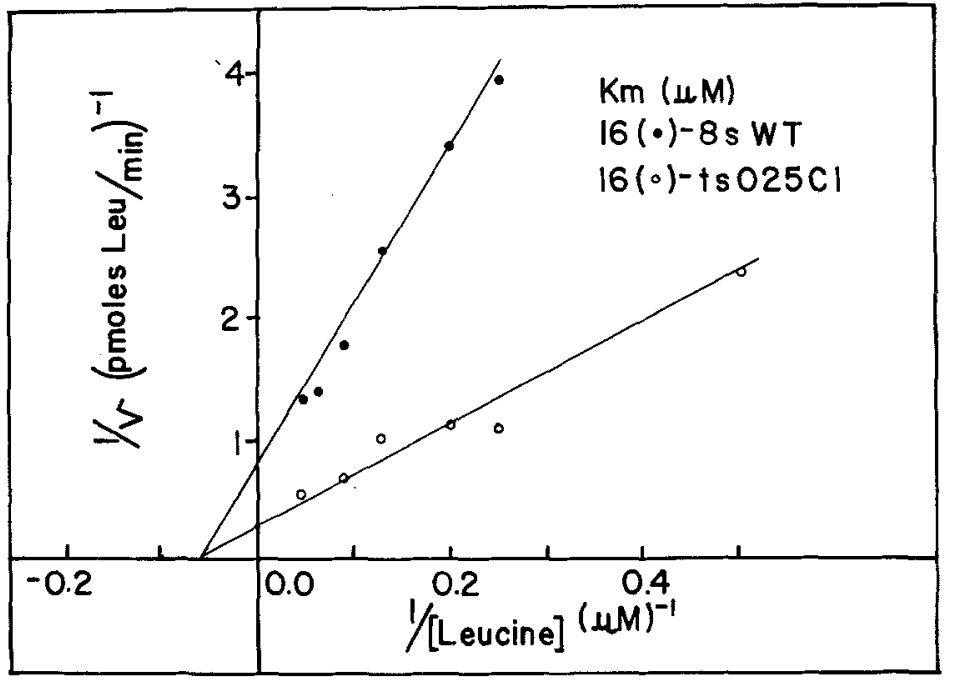

Fig. 3. Leucine Michaelis constants of wild-type $8 \mathrm{~S}$ LeuRS and mutant $t s 025 \mathrm{Cl}$ 8 S LeuRS. 
thermostable than the $8 \mathrm{~S}$ form of the enzyme (Klekamp et al., 1981). The fact that $t s 025 \mathrm{Cl}$ contains none of these thermostable forms and low levels of $8 \mathrm{~S}$ can readily account for the temperature-sensitive phenotype. This is further supported by the phenotypic protective effect on the parent of $t s 025 \mathrm{Cl}$, $t s 025$, of high levels of leucine (Thompson et al., 1975), which we have shown is seen in mutants when no corresponding high molecular weight synthetase complexes are present (Klekamp et al., 1981; Pahuski et al., 1983). Since the parent of $t s 025 \mathrm{Cl}, t s 025$, does not genetically complement the mutant $t s \mathrm{H} 1$, it is most likely that the LeuRS enzyme in $t s 025 \mathrm{Cl}$ is altered, and indeed the gene locus for LeuRS was genetically mapped to human chromosome 5 (Giles et al., 1980). This is further supported by mapping studies with human $\times$ $t s \mathrm{Hl}$ hybrids which also defined the LeuRS locus at human chromosome 5 (Dana and Wasmuth, 1982).

What, then, is the molecular basis for the $t s 025 \mathrm{Cl}$ mutation? The evidence argues that most likely $t s 025 \mathrm{Cl}$ LeuRS contains as alteration in the LeuRS structural protein itself which does not affect the catalytic activity or structure but does prevent the formation of complexes. It now becomes important to analyze the LeuRS of the human $\times$ hamster $(t s 025 \mathrm{Cl})$ temperature-resistant hybrid to determine if human gene products other than LeuRS are stabilizing a CHO LeuRS complex in the hybrid. This possibility is further supported by the observation that human gene products can enter hamster multicomponent complexes in interspecific hybrids (Dana and Wasmuth, 1982). A number of possible combinations of human hamster LeuRS and complex proteins could exist to confer the temperature-resistant phenotype of the hybrid which are amenable to study with the $t s 025 \mathrm{Cl}$ mutant.

\section{ACKNOWLEDGMENTS}

We thank Lynn Isoye, Dorthy Ling, and Wiley Taylor for technical assistance.

\section{REFERENCES}

Bandyopadhyay, A., and Deutscher, M. (1971). Complex of aminoacyltransfer RNA synthetase. J. Mol. Biol. 60:113.

Dana, S., and Wasmuth, J. (1982). Linkage of the leuS, emt $\mathbf{S}$ and chr genes on chromosome 5 in humans and expression of human genes encoding protein synthetic components in humanChinese hamster hybrids. Somat. Cell Genet. 8:245.

Dingman, C. (1972). A convenient program for the rapid calculation of sedimentation coefficients in linear salt or sucrose gradients. Anal. Biochem. 47:124.

Farber, R., and Deutscher, M. (1976). Physiological and biochemical properties of temperature sensitive leucyl-tRNA synthetase mutant $t s \mathrm{Hl}$ and revertants of Chinese hamster cells. Somat. Cell Genet. 2:509.

Giles, R., Schimizu, N., and Ruddle, F. (1980). Assignment of a human genetic locus to 
chromosome 5 which corrects the heat sensitive lesion associated with reduced leucyl-tRNA synthetase activity in $t s 025 \mathrm{Cl}$ Chinese hamster cells. Somat. Cell Genet. 6:667.

Haars, L., Hampel, A., and Thompson, L. (1976). Altered leucyl-transfer RNA synthetase from a mammalian cell mutant. Biochim. Biophys. Acta 454:493.

Hampel, A., Ritter, P., and Enger, M. (1978). A physically altered leucyl-tRNA synthetase complex in a CHO cell mutant. Nature 276:844.

Klekamp, M., Pahuski, E., and Hampel, A. (1981). Reformation of leucyl-tRNA synthetase complexes in revertants from CHO mutants $t s \mathrm{Hl}$. Somat. Cell Genet. 7:725.

Lowry, O., Rosebrough, M., Farr, A., and Randall, R. (1951). Protein measurement with the folin phenol reagent. J. Biol. Chem. 193:265.

McBurney, M., and Whitmore, G. (1975). Isolation and biochemical characterization of folate deficient mutants of Chinese hamster cells. Cell 2:173.

Pahuski, E., Klekamp, M., Condon, T., and Hampel, A., (1983). Altered aminoacyl-tRNA synthetase complexes in CHO cells. J. Cell. Physiol. 114:82.

Ritter, P., Enger, M., and Hampel, A. (1976). Aminoacyl-tRNA synthetase in normal, mutant, and arrested CHO cells. In Fishman, W. H., and Sell, S. (eds), Onco-Developmental Gene Expression, Academic Press, New York, p. 47.

Ritter, P., Enger, M., and Hampel, A. (1979). Characterization of postribosomal aminoacyltRNA synthetase in cultured Chinese hamster ovary cells. Biochim. Biophys. Acta 562:377.

Schimmel, P., and Soll, D. (1979). Aminoacyl-tRNA synthetases: General features and recognition of transfer RNAs. Annu. Rev. Biochem. 48:601.

Thompson, L., Harkins, J., and Stanners, C. (1973). A mammalian cell mutant with a temperature sensitive leucyl-tRNA synthetase. Proc. Natl. Acad. Sci. USA 70:3094.

Thompson, L., Harkins, J., and Siminovitch, L. (1975). Selection by $\left({ }^{3} H\right)$ amino acids of CHO cell mutants with altered leucyl and asparagyl-transfer RNA synthetases. Somat. Cell Genet. 1:187.

Thompson, L., Lofgren, D., and Adair, G. (1977). Cell mutants for arginyl-, asparagyl-, glutaminyl-, histidyl-, and methionyl-transfer RNA synthetases: Identification and initial characterization. Cell 11:157.

Wilkinson, G. N. (1961). Statistical methods in enzyme kinetics. Biochem. J. 80:324. 